\title{
Electron cloud effects on beam evolution in a circular accelerator
}

\author{
G. Rumolo, ${ }^{1}$ A. Z. Ghalam, ${ }^{2}$ T. Katsouleas, ${ }^{2}$ C. K. Huang, ${ }^{3}$ V. K. Decyk,${ }^{3}$ C. Ren, ${ }^{3}$ W. B. Mori, ${ }^{3}$ \\ F. Zimmermann, ${ }^{1}$ and F. Ruggiero ${ }^{1}$ \\ ${ }^{1}$ CERN, Geneva, Switzerland \\ ${ }^{2}$ University of Southern California, Los Angeles, California, USA \\ ${ }^{3}$ University of California at Los Angeles, Los Angeles, California, USA
}

(Received 17 March 2003; published 15 August 2003)

\begin{abstract}
The interaction between a low-density electron cloud in a circular particle accelerator with a circulating charged particle beam is considered. The particle beam's space charge attracts the cloud, enhancing the cloud density near the beam axis. It is shown that this enhanced charge and the image charges associated with the cloud charge and the conducting wall of the accelerator may have important consequences for the dynamics of the beam propagation. The tune shift due to the electron cloud is obtained analytically and compared to a new numerical model (QUICKPIC) that is described here. Sample numerical results are presented and their significance for current and planned experiments is discussed.
\end{abstract}

DOI: 10.1103/PhysRevSTAB.6.081002

PACS numbers: 29.27.Bd, 29.27.Eg, 52.65.Rr, 52.27.Jt

\section{INTRODUCTION}

Electron clouds have been shown to be associated with limitations in particle accelerators performance in several of the world's largest circular proton and positron machines [1]. Electrons accumulate in the vacuum chamber where a positively charged bunched particle beam propagates because of a multipacting process which involves primary electron generation (e.g., from residual gas ionization or from photoemission at the inner pipe wall due to synchrotron light) and their multiplication through secondary emission at the wall [2]. If the average secondary emission yield of the chamber material is greater than unity, the primary electrons accelerated in the field of the passing bunch can reach the pipe wall with a high enough energy to produce more electrons. The number of electrons in the pipe will then grow until an equilibrium is reached between the production rate (dominated by secondary emission) and the loss rate progressively increased by low energy electrons quickly repelled to the wall because of the high space charge field from the cloud itself. The presence of an electron cloud inside the beam chamber can deteriorate the vacuum $[3,4]$, causing interference on the electrodes of beam pickup monitors [2] and make the beam unstable via bunch-to-bunch or head-tail coupling [5-8].

Although a number of beam-cloud interaction models that can satisfactorily explain many of the observations have been developed [9-15], the exact dynamics that leads to beam degradation (e.g., emittance growth, beam loss) is still under study for a more detailed understanding. In particular, previous models have been limited in that they treat the cloud in an impulse approximation [12] (giving a single kick per turn) Also, the conducting boundary of the pipe (and hence image forces of the cloud and beam charge) has not been included in the treatment of the dynamics of the beam in these models although it may have been considered in the cloud buildup process [16-18]. In addition, there are discrepancies between the analytic models to date and the experimentally observed tune shifts.

In this paper we apply techniques from plasma physics and plasma wake field accelerator models to the study of the non-neutral plasma-beam interaction. We describe analytic and numerical models for the interaction between a positively charged beam and an electron cloud in a circular accelerator. Our model takes into account the effect of cloud image charges from the conducting beam pipe on the beam dynamics for the first time. We show that (i) the cloud space charge force is the dominant force in contributing to the coherent tune shift. (ii) The cloud compression on the axis of the beam along with its image charges on the conducting walls of the accelerator may not contribute to the coherent tune shift, but instead, play an important role in configuring the steady state beam dynamics. The simulation model is adapted from a particle in cell (PIC) plasma model of wake field accelerators in the quasistatic approximation [19]. The full description of the simulation tool and the modifications to adapt it to circular accelerator environment are given in Sec. II. The forces on the beam by the electron cloud and the cloud and beam image charges in the conducting wall are analyzed in Sec. III. In Sec. IV, the interaction between these forces (we study how these forces interact with each other) and their effects on the dynamics of the beam are studied. Section V is devoted to the tune shift due to the electron cloud. An analytic expression for the tune shift is derived and compared to the PIC simulation results. Finally, emittance growth and beam instabilities due to the beam-cloud interactions are discussed in Sec. VI. 


\section{DESCRIPTION OF THE SIMULATION MODEL (QUICKPIC) WITH THE SYNCHROBETATRON OSCILLATIONS}

QUICKPIC is a new 3D PIC code based on the quasistatic or frozen field approximation [19-21]. This approximation is specifically useful for studying wakes. It requires that the beam does not evolve significantly on the time scale that it takes the beam to pass by plasma particles, or in other words, $\beta \gg \sigma_{z}$, where $\beta$ is the average beta function and $\sigma_{z}$ is the axial length of the beam viewed in the lab frame. This is typically well satisfied in the models discussed in this paper. The basic equations for QUICKPIC follow from the wave equations for $A$ and $\phi$ in the Lorentz gauge as illustrated in the box in Fig. 1.

The quasistatic approximation assumes that the wakes are functions of $z$-ct only so that the full set of Maxwell's equations reduces to equations for the wake potentials $\varphi$ and $\Psi=\varphi-A_{\|}$that involve only solving 2D Poisson equations. The QUICKPIC cycle is illustrated in Fig. 2. The Poisson equations are solved on a $2 \mathrm{D}$ slab of plasma (using a well-established bounded 2D PIC code BEPS [22] as a subroutine) with conducting boundary conditions. The longitudinal motion of the cloud electrons and $V \times B$ force and the $B$ field terms arising from their motion are neglected in the present implementation. This is typically valid as long as the spot size of the beam is significantly less than the collisionless skin depth of the cloud, $c / \omega_{p}$, where $\omega_{p}$ is the plasma frequency associated with the cloud electrons, and the cloud particle velocities remain nonrelativistic. Both of these assumptions are valid here. The full quasistatic equation including relativistic and electromagnetic corrections is currently being implemented and will be available for future studies. Having assumed that the beam particles are moving relativistically and the cloud particles are nonrelativistic, the contribution of the longitudinal cloud particle's current density to the total current density is negligible although no assumption is made for the relative

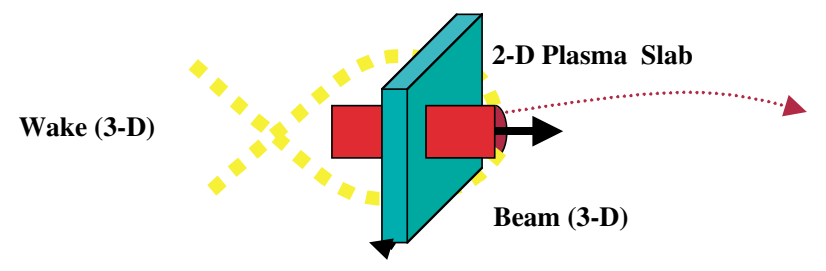

FIG. 2. (Color) QUICKPIC cycle. A 2D Poisson solver is used to calculate potentials and update positions and velocities in the plasma slab. After the slab is stepped through the beam, the stored potentials $\Psi$ and $\varphi$ are used to push the 3D beam.

magnitude of the cloud and beam charge densities. No electron production is currently implemented in the code and cloud electrons are elastically reflected from the conducting walls of the chamber. The cloud is assumed to be generated by the preceding bunches and is assumed to be initially uniform and cold.

The wakes are stored and used to update the plasma in the slab and the slab is then pushed back a small step through the beam. After transiting the beam, the stored values of $\psi$ are used to find the force on the beam (treated as a 3D PIC model) and it is pushed through a large step (of the order of $\beta / 30$ ). In a standard electromagnetic PIC model the time step, $\Delta t$, is nearly cell size divided by the speed of light, $\Delta z / c$. For this quasistatic code, the time step is $\beta / 30$ leading to 2 or 3 orders of magnitude fewer time steps and an identical savings of computing time. The code is highly optimized on a single processor and both the 3D outer layer and the 2D inner layer of the code are fully parallelized to allow domain decomposition along $z$ and $y$, respectively.

As mentioned, the code is used for modeling plasma wake field accelerators. In order to enable QUICKPIC to simulate a bunch in a circular accelerator, some extra features were added to correctly model the bunch evolution. In particular, betatron and synchrotron oscillations of the beam particles are introduced. These oscillations

$$
\begin{aligned}
& \left(\frac{1}{c^{2}} \frac{\partial^{2}}{\partial t^{2}}-\nabla^{2}\right) \mathbf{A}=\frac{4 \pi}{c} \mathbf{j} \\
& \left(\frac{1}{c^{2}} \frac{\partial^{2}}{\partial t^{2}}-\nabla^{2}\right) \phi=4 \pi \rho \\
& \mathbf{j}=\mathbf{j}_{b}+\mathbf{j}_{e} \approx \mathbf{j}_{b}=c \rho_{b} \hat{z} \\
& \Psi=\phi-A_{/ /}
\end{aligned}
$$

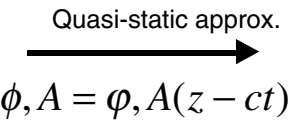$$
-\nabla_{\perp}^{2} \mathbf{A}=\frac{4 \pi}{c} \mathbf{j}
$$$$
-\nabla_{\perp}^{2} \phi=4 \pi \rho
$$

Local-- $\phi, A$ at any z-slice depend only on $\rho, j$ at that slice!

\begin{tabular}{cc}
\hline $\begin{array}{c}\text { Forces : } \\
\text { plasma : }\end{array}$ & $F_{e \perp}=-e \nabla_{\perp} \phi$ \\
beam : & $F_{b \perp}=-e \nabla_{\perp} \Psi$ \\
\hline
\end{tabular}

FIG. 1. (Color) Quasistatic or frozen field approximation used in QUICKPIC. 
are due to the external fields of the magnets and rf power in the accelerator. Under the effect of these forces, individual particles (and the bunch as a whole, if off centered) execute oscillations in all three spatial coordinates. The frequency of the transverse oscillation of the single particle has been made dependent upon its longitudinal momentum offset to take into account also chromatic effects that seem to play an important role in the unstable evolution of the beam [12]. The equations of motion of a single beam particle in transverse and longitudinal directions implemented in QUICKPIC are as follows:

$$
\begin{aligned}
& \frac{d^{2} x}{d t^{2}}+\left[Q_{x}+\Delta Q_{x}\left(\frac{\delta p}{p_{0}}\right)\right]^{2} \omega_{0}^{2} x=\frac{1}{m_{p} \gamma} F_{\mathrm{cl}_{x}}, \\
& \frac{d^{2} y}{d t^{2}}+\left[Q_{y}+\Delta Q_{y}\left(\frac{\delta p}{p_{0}}\right)\right]^{2} \omega_{0}^{2} y=\frac{1}{m_{p} \gamma} F_{\mathrm{cl}_{y}},
\end{aligned}
$$

and

$$
\left\{\begin{array}{c}
\frac{d z}{d t}=-\eta c\left(\frac{\delta p}{p_{0}}\right), \\
\frac{d}{d t}\left(\frac{\delta p}{p_{0}}\right)=\frac{Q_{s}^{2} \omega_{0}^{2}}{\eta c} z+\frac{1}{p_{0}} F_{\mathrm{cl}_{z}} .
\end{array}\right.
$$

In these equations $Q_{x}$ and $Q_{y}$ are, respectively, the horizontal and vertical tunes; $\Delta Q_{x}$ and $\Delta Q_{y}$ represent the chromatic shifts proportional to the particle relative momentum offset $\left(\delta p / p_{0}\right) ; \omega_{0}$ is the angular revolution frequency of the beam in the circular accelerator (which for ultrarelativistic beams can be written as $c / R_{0}, R_{0}$ being the average machine radius); $F_{\mathrm{cl}}=$ $\left(F_{\mathrm{cl}_{x}}, F_{\mathrm{cl}_{y}}, F_{\mathrm{cl}_{z}}\right)$ is the total force exerted by the cloud on each beam particle; $p_{0}$ is the nominal particle momentum; $\gamma$ is the relativistic factor; $\eta$ is the slippage factor, which can be positive if the machine is operating above transition energy, thus causing more energetic particles to move backwards within the bucket and vice versa. For simplicity, in our model we have replaced the alternating gradient focusing, on which most of the real machines are based, with constant and uniform focusing in both transverse directions. The longitudinal equations of motion represent in linearized form the rf bucket that focuses the beam longitudinally.

Unless otherwise specified, the simulation parameters used in the simulation throughout the entire paper are matched to CERN Super Proton Storage (CERN-SPS) parameters. These parameters are summarized in Table I.

The simulation box is $40 \mathrm{~mm}$ in the vertical direction, $80 \mathrm{~mm}$ in the horizontal, and $2.5 \mathrm{~m}$ in the longitudinal direction. There are 64-128 cells in each direction and 4-8 particles (electron cloud particles) in each cell; the total number of beam particles in the simulation box is 524288 .
TABLE I. SPS parameters used in the simulations.

\begin{tabular}{lc}
\hline \hline rms horizontal spot size $(\mathrm{mm})$ & 2 \\
rms vertical spot size $(\mathrm{mm})$ & 2 \\
rms bunch length $(\mathrm{cm})$ & 30 \\
Horizontal box size $(\mathrm{mm})$ & 80 \\
Vertical box size $(\mathrm{mm})$ & 40 \\
Bunch population & $10^{11}$ \\
Horizontal emittance $(\mu \mathrm{m})$ & 0.1 \\
Vertical emittance $(\mu \mathrm{m})$ & 0.1 \\
Momentum spread & $2.48 \times 10^{-3}$ \\
Beam momentum $(\mathrm{GeV} / \mathrm{c})$ & 26 \\
Circumference $(\mathrm{km})$ & 6.9 \\
Horizontal betatron tune & 26.22 \\
Vertical betatron tune & 26.18 \\
Synchrotron tune & 0.005 \\
Electron cloud density $\left(\mathrm{cm}^{-3}\right)$ & $10^{6}-10^{7}$ \\
\hline \hline
\end{tabular}

\section{FORCES ACTING ON THE BEAM}

As the model equations indicate, the fields on each longitudinal piece of the beam and cloud come from 2D Poisson equations. So, all forces can be viewed as if from long line charge or current densities. There are three space charge forces to consider. First, there are the direct image charges of the beam with line charge density and line current equal and opposite to the beam. For relativistic beams, the electric and magnetic forces from these images cancel to order $1 / \gamma^{2}$ and as we will show later they can be neglected. Second, there is a direct force from the cloud on the beam. When the cloud is unperturbed (uniform density) this gives a restoring force proportional to the electric field:

$$
E_{d_{\mathrm{c}} \mathrm{l}} \approx \frac{n_{\mathrm{cl}} e y}{2 \varepsilon_{0}} .
$$

When a positively charged bunch passes through an electron cloud it sucks in the cloud towards its axis, creating cloud compression near the axis of the beam. Figure 3 shows QUICKPIC results for a positively charged beam passing through an electron cloud and the corresponding cloud density at early times. Each image is a color contour plot of the density for an $x$ - $z$ plane with $y$ at the center of the beam. As can be seen from the cloud density figure, there is a high concentration of cloud close to the beam axis, increasing toward the tail of the beam. The pinched cloud exerts a force to an unperturbed beam and focuses the beam toward its own axis.

The situation becomes more complicated when the beam is tilted as in Fig. 4. In this case, the compressed cloud is not aligned perfectly with the beam axis. As shown in Fig. 4, this creates an electric field and a force on the beam tail in a direction that pulls the tail back to the not-tilted axis. Approximating the pinched cloud as a uniform line density much longer than the bunch length, and also assuming the tilt amount is less than the cloud 

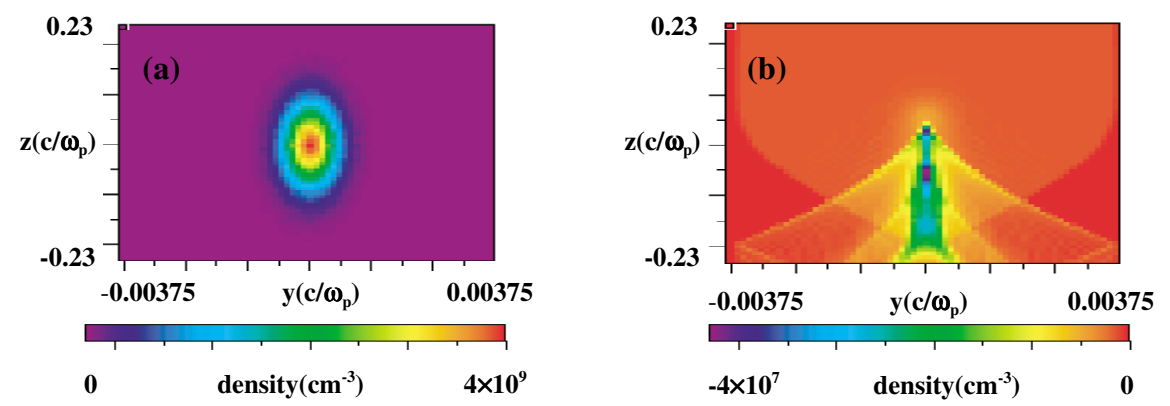

FIG. 3. (Color) (a) Positively charged beam density. (b) The corresponding cloud density. Cloud compression on the axis of the beam is evidenced from this figure. The color bars show the beam and cloud densities. The unperturbed (uniform density) cloud density is $10^{6} \mathrm{~cm}^{-3}$ and $c / \omega_{p}$ is $5.3 \mathrm{~m}$.

compression cross section, the electric field due to the line density at distance $y$ from the axis can be written as

$$
E_{\mathrm{cl}_{y}} \approx-\frac{\lambda_{\mathrm{cl}} y}{2 \varepsilon_{0} A} .
$$

Here, $A$ is the cross section area of the pinched cloud near the axis of the beam and $\lambda_{\mathrm{cl}}$ is the line charge density of the compressed cloud.

When we have conducting boundary conditions, there is yet another restoring force acting on the displaced beam. The pinched cloud on the axis of the beam produces its own image charges of like sign of the beam in a conducting pipe and hence in a direction as to restore the beam to the pipe axis [20]. These images are not moving relativistically and hence there is no corresponding cancellation of their effect by the magnetic forces as was the case for the beam images.

To quantify the image cloud forces acting on the beam we consider a negative line charge density $-e \lambda_{\mathrm{cl}}$ located at $a / 2, b / 2+y$ inside a rectangular box of dimensions $a$ and $b$, as shown in Fig. 5 .

This charge models the accumulation of electrons around a slightly displaced beam. Its images will act

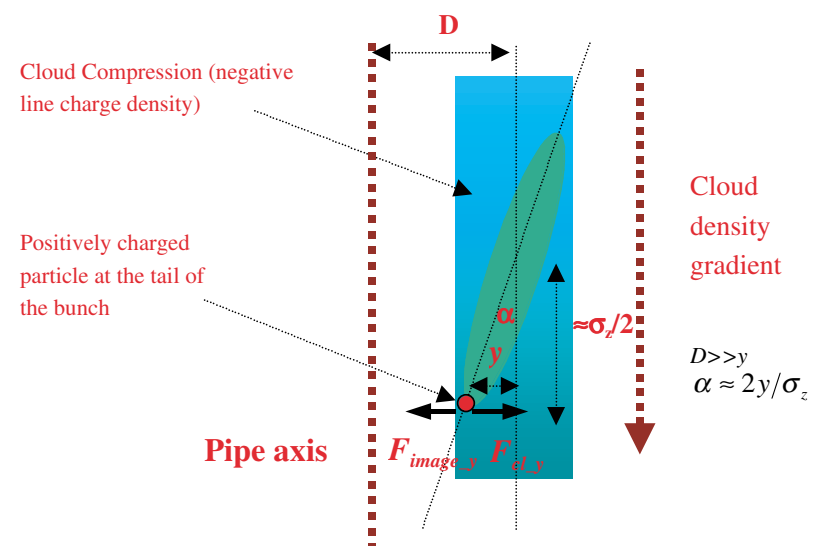

FIG. 4. (Color) A cartoon showing a tilted beam and the electric field on the tail of the beam due to cloud compression on the axis. The beam is moving up. back on the beam. We assume that the displacement $y$ of this charge from the center of the box is small compared to the box vertical size $b$. Neglecting the electric field from the image charges in the vertical conducting pipe $(b \ll a)$, the total electric field from the images evaluated at the location where the source charge is can be written as

$$
\begin{gathered}
E_{\text {image }_{y}}(y)=\frac{\lambda_{\mathrm{cl}} e}{2 \pi \varepsilon_{0}}( \\
-\frac{1}{b-2 y}+\frac{1}{b+2 y}-\frac{1}{3 b-2 y} \\
\left.+\frac{1}{3 b+2 y}-\cdots\right) .
\end{gathered}
$$

For $y \ll b$, the field becomes

$$
E_{\text {image }_{y}}(y)=-\frac{2 y \lambda_{\mathrm{cl}} e}{\pi \varepsilon_{0} b^{2}} \sum_{n \text { odd }}\left(\frac{1}{n^{2}}\right)=-\frac{\pi \lambda_{\mathrm{cl}} e}{4 \varepsilon_{0} b^{2}} y .
$$

Each bunch particle (proton or positron) will feel a focusing force given by $F_{\text {image }_{y}}(y)=e E_{\text {image }_{y}}(y)$.

The cloud line charge $\lambda_{\mathrm{cl}}$ can be expressed as

$$
\lambda_{\mathrm{cl}}=H_{\mathrm{cl}}(z) n_{\mathrm{cl}} e A,
$$

where $A$ is the cross sectional area of the cloud density enhancement on the beam axis, and $H_{\mathrm{cl}}$ is the cloud compression enhancement factor. $H_{\mathrm{cl}}$ can be estimated from simulations or an analytic expression found in the

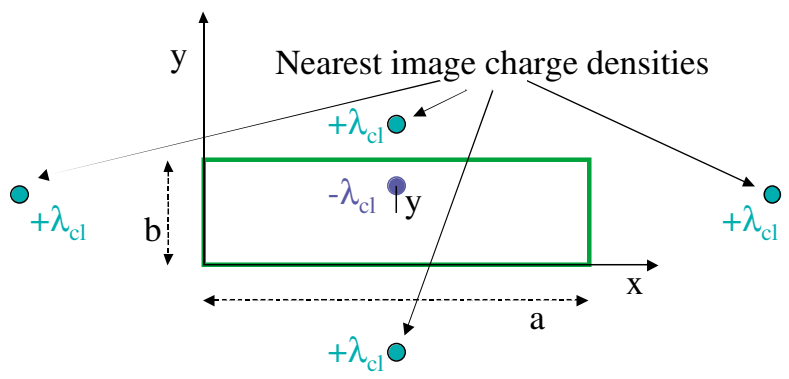

FIG. 5. (Color) Electrons attracted by a vertically displaced beam are modeled as a line density whose images with respect to the perfectly conducting wall act back on the beam. The green box is the conducting cavity. 
Appendix:

$$
H_{\mathrm{cl}} A=2 \pi \sigma_{r}^{2}\left(\frac{\omega_{\mathrm{pb}} \sigma_{z}}{c}\right)^{2}
$$

where $\omega_{\mathrm{pb}}$ is the electron plasma frequency associated with the beam density.

In the next section, we give a qualitative description of beam dynamics based on the interaction between the forces on the beam.

\section{EFFECT OF CLOUD AND CLOUD IMAGE FORCES ON BEAM DYNAMICS}

Because of the nonlinearities involved in the problem, describing the interaction between the three forces on the beam due to the cloud by analytic expressions is complex; however, a simple description of the scenario is as follows:

Imagine a beam off centered from the axis of the pipe but not tilted. The beam experiences a force due to the unperturbed space charge of the cloud that deflects it toward the axis and therefore shifts the betatron tune. In addition, the cloud compression exerts an added force that focuses but does not displace the beam (since the beam is not tilted). The forces due to image charges though, exert a force $\left(F_{\text {image }_{y}}\right.$ in Fig. 4$)$ on the beam towards the axis of the pipe. This force tends to be larger at the tail than it is at the head due to cloud compression near the tail. So, the tail tends to dephase from the head causing the beam to tilt. As soon as the beam tilts, the force due to direct cloud compression ( $F_{\mathrm{cl}_{y}}$ in Fig. 4$)$ acts on the tail trying to pull it back towards the beam axis as shown in Fig. 4. So, there is an equilibrium angle at which the force due to the direct cloud and its images cancel each other at the tail. A lower bound on the tilt angle could be found by setting the forces due to image cloud compression and the direct cloud compression [Eqs. (2) and (3) with $y$ replaced by $D-y \approx$ $D$ in Eq. (3)] to be equal. Using Eq. (2) as a force due to direct cloud compression implies that the compression is assumed to be completely parallel to the axis of the pipe, but, in reality cloud compression lies somewhere between the axis of the beam and the axis of the pipe. Thus Eq. (2) sets up an upper limit to the force from the pinched cloud and therefore sets up a lower limit for the equilibrium tilt angle:

$$
\alpha>\frac{\pi^{2} \sigma_{x} \sigma_{y} D}{b^{2} \sigma_{z}}
$$

where, $D$ is the displacement amount and $b$ is the vertical pipe size. In deriving this equation, the cross section area of the cloud density compression in Eq. (2) is approximated as $\pi \sigma_{x} \sigma_{y}$.

Before proceeding further we make two side comments. (1) In this discussion, we have neglected the image forces due to the unperturbed cloud. Numerically we found that these contribute a correction of less than $20 \%$. (2) We are typically considering a regime in which the bunch is sufficiently short or the box size is sufficiently large such that electrons are not depleted on either side of the beam. From the Appendix, Eq. (A3), this is $\left(\omega_{\mathrm{pb}} \sigma_{z} / c\right) \leq\left(b / \sigma_{r}\right)$.

To test the physical picture explained here, we look at the force on the tail of a beam using QUICKPIC simulations. Figure 6 shows the force at the tail of a beam versus tilt angle for different beam displacements. As can be seen, the force on the tail crosses zero at some tilt angle for each displacement amount. This angle is the equilibrium angle. The more the beam is deflected the bigger the image charge forces on the beam would be and therefore the bigger the equilibrium angle is. From the figure we see, for example, for the displacement of $5 \mathrm{~mm}$, the equilibrium angle is around $5^{\circ}$; estimating this angle by (6) will give the tilt amount of about $0.2^{\circ}$.

As a comparison to previous models [9-12] which did not include conducting boundaries [15-19], we show as a dashed curve in Fig. 6 the force on the beam, displaced $7.5 \mathrm{~mm}$ from the center, but with periodic boundary conditions. As seen this force is quite different from

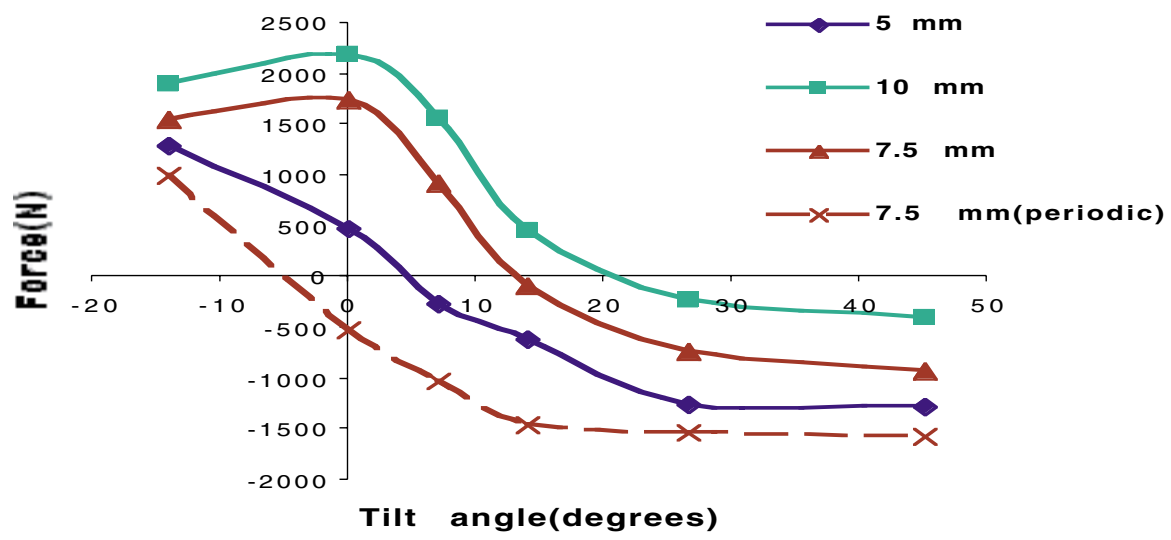

FIG. 6. (Color) Forces on the beam as a function of the tilt angle for different displacement amounts. 

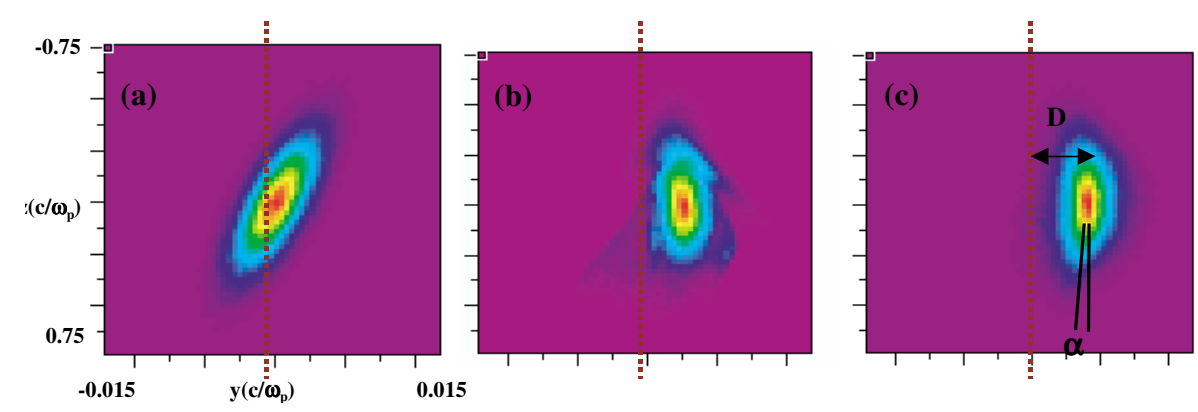

FIG. 7. (Color) Three snapshots of beam evolution over CERN-SPS. (a) At $t=0$; (b) at $t=136 \mu$ s (6 turns); (c) at $t=0.8$ ms (35 turns). $c / \omega_{p}$ for all the snapshots is $1.67 \mathrm{~m}$.

the corresponding force on the beam with conducting boundary conditions. This difference between the two cases shows the importance of proper boundary conditions of the cloud image forces on the beam.

To verify the above argument we look at the long-term propagation of a beam along CERN-SPS. The physical parameters chosen in the simulation are matched to the CERN-SPS physical parameters. The cloud density is chosen to be $10^{7} \mathrm{~cm}^{-3}$. As an initial perturbation, the beam is tilted but on axis at the beginning of the simulation (with a slope of $1 \sigma_{x}$ per $1 \sigma_{z}$ ). Figure 7 shows three snapshots of the beam during the simulation. At the beginning of the simulation [Fig. 7(a)], there is a force due to direct cloud compression acting on the beam. This force is much greater at the tail than it is at the head due to the fact that charge compression reached its maximum close to the tail of the beam. Neglecting the force on the head compared to the tail, we can say that the force tends to line up the tail with the head later in time. Figure 7(b) shows the beam density after six turns $(36 \mathrm{~km})$. As can be seen, the beam is not tilted anymore but it is deflected from the center due to the fact that the tail has lined up with the head. Since the head was initially off axis, there is an overall displacement to the beam. At still later times, the beam develops a slight equilibrium tilt angle described in the previous section. This is shown in Fig. 7(c) which is the beam density after 35 turns around the accelerator ring.

The amount of displacement from Fig. 7 is about $6.8 \mathrm{~mm}$ and the tilt angle amounts to $9^{\circ}$. This amount of tilt is consistent with that predicted by Fig. 6 which is between $5^{\circ}$ and $10^{\circ}$ for $6.8 \mathrm{~mm}$ of displacement.

\section{EFFECTS OF CLOUD AND CLOUD IMAGE FORCES ON THE TUNE SHIFT}

As mentioned earlier, there is a force due to unperturbed cloud space charge on the beam. Assuming that the bunch goes through an initial uniformly distributed electron cloud, the head of the bunch feels the field (1) and consequently experiences a tune shift:

$$
\Delta Q_{y}=\frac{e^{2} n_{\mathrm{cl}} R_{0}^{2}}{4 \varepsilon_{0} m_{p} c^{2} Q_{y} \gamma}
$$

Moreover, the study in [10] suggests that in open space, if the cloud size is much larger than the beam size, the coherent tune shift can be evaluated by naively using this expression applied to the whole bunch even in the dynamical regime while the electron cloud gets distorted by the passing bunch. This was a result of numerical simulations and was explained as an effect from electrons at large amplitudes, which perform slow nonlinear oscillations around the bunch and hence give rise to a static force acting on the bunch at any time.

We may expect the forces due to direct cloud compression and its images to be inconsequential to the tune shift or tune spread as they tend to cancel each other at steady state as shown before. To test this, we examine the tune shift and spread using QUICKPIC. Figure 8 shows the results for vertical tune lines for $10^{6}$ and $10^{7} \mathrm{~cm}^{-3}$ cloud densities, the two representative values of what is expected at the CERN-SPS. The tune line for no cloud case is also depicted for the sake of comparison.

The tune lines are obtained by taking the fast-Fourier transform of the beam centroid motion over 150 turns of beam propagation. From the figure the tune shift for $10^{6} \mathrm{~cm}^{-3}$ cloud density is 0.007 while that of $10^{7} \mathrm{~cm}^{-3}$ cloud density is 0.077 which is almost 10 times as big as the low-density case. This tune shift and the linear proportionality of the tune shift with density are consistent with Eq. (7), the result for simple unperturbed cloud.

There is relatively little tune spreading observed in the tune lines of Fig. 8 (i.e., tune spread is much less than the tune shift), suggesting that the cloud compression and its image forces, although large contribute little to tune shift or tune spread of the accelerator. We note, however, that at this point our model does not include gradients in the cloud density along the ring (e.g., gradients in magnetic field) and that these variations may disrupt the equilibrium scenario described in the previous section and may lead to tune spread. This is a subject for further work. 


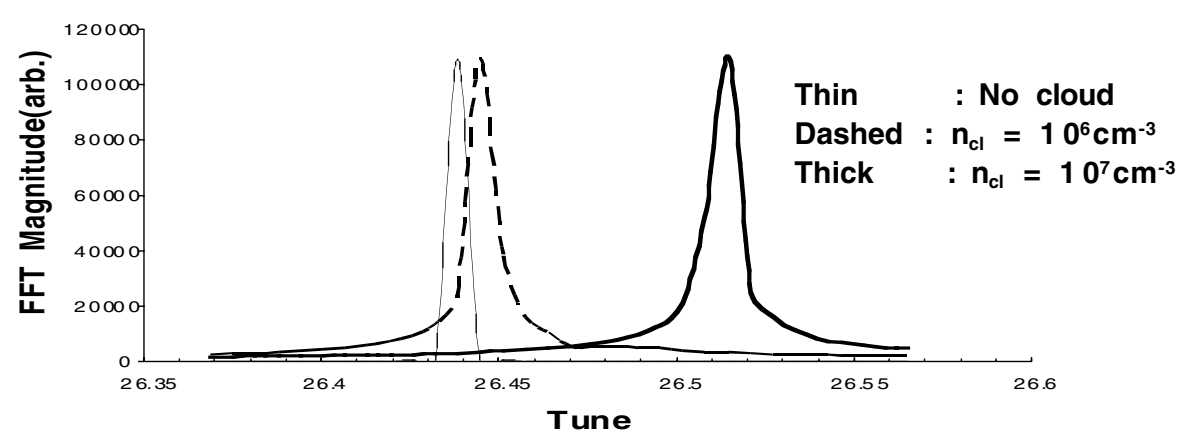

FIG. 8. Vertical tune for different cloud densities.

\section{EFFECTS OF ELECTRON CLOUD ON BEAM INSTABILITY}

Next, the effects of the electron cloud on the emittance growth of the beam are studied. The simulations are performed based on the CERN- SPS parameters for 70 turns of the SPS ring. The beam is initially untilted but off axis $1 \mathrm{~mm}$ in the vertical direction. Over this length, there is no emittance growth in our model in the absence of electron cloud and negligible emittance growth for cloud densities up to $10^{6} \mathrm{~cm}^{-3}$ [Fig. 9(a)]. This is a typical value for cloud density obtained from the simulations of electron cloud buildup for the CERNSPS ring [17]. However, at cloud densities 10 times higher, the emittance growth of the beam becomes apparent. Figure 9(b) shows the spot size of the beam in this high cloud density. As seen from the figure, the spot size grows $50 \%$ after 70 turns. Figure 9(c) shows that the beam centroid oscillation also grows.

Figure 10 shows three snapshots of the beam after 18 turns over half a betatron period for the high cloud density case. It is interesting to compare Fig. 10(c) with Fig. 7(c) where the beam was initially tilted. We see in both cases the beam shows similar behavior: oscillating around the axis of the pipe with the tail slightly tilted toward the center line with respect to the head toward the axis. This shows that no matter how the beam is initially perturbed, the beam ends up having a similar long-term dynamic.

Work is in progress to further study the longer term evolution of the beam (over a few hundred turns).

\section{DISCUSSION}

We have presented new analytic and numerical models for the dynamics of electron clouds and beams in a circular accelerator. Our new parallelized model allows continuous modeling (i.e., resolution less than beta function) of the beam along the accelerator and includes the effects of image forces of the cloud on the beam dynamics for the first time. Results of the model for tune shift and tune spread are in fair agreement with previous work. However the emittance growth seen in QUICKPIC is a factor of a few times slower than that seen in other models [12] for which the cloud was treated as a single
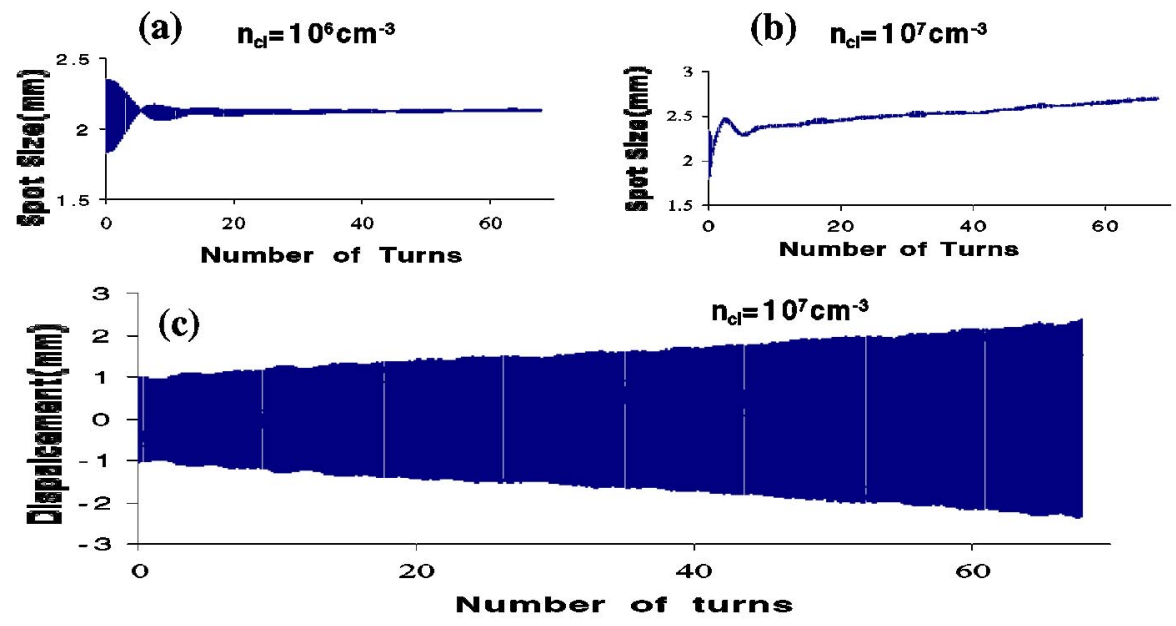

FIG. 9. (Color) QUICKPIC simulation of beam propagation over 70 turns of CERN-SPS (a) and (b) spot sizes for cloud densities $10^{6}$ and $10^{7} \mathrm{~cm}^{-3}$, respectively. Some oscillation is observed initially in (a) that is because the beam is not matched initially. (c) Beam centroid in the vertical plane for $10^{7} \mathrm{~cm}^{-3}$ of cloud density. 


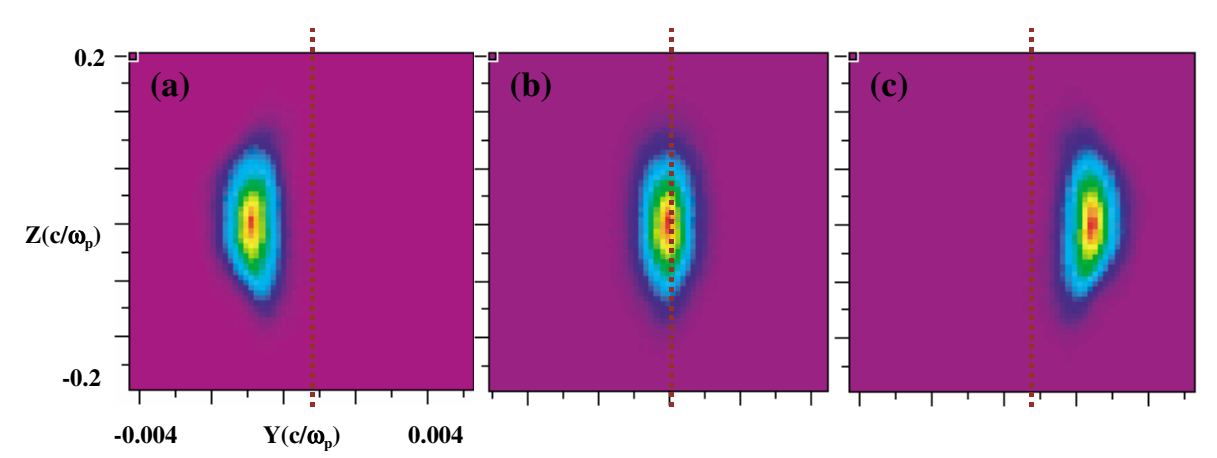

FIG. 10. (Color) Three snapshots of the beam over the CERN-SPS ring. (a) At $t_{0}=0.4 \mathrm{~ms}\left(18\right.$ turns); (b) at $t_{1}=t_{0}+T_{B} / 4 ;$ (c) at $t 2=t_{0}+T_{B} / 2$, where $T_{B}$ is the nominal betatron period $(\approx 0.9 \mu \mathrm{s}) . c / \omega_{p}$ for all the snapshots is $1.67 \mathrm{~m}$.

slab at one point in the ring. This suggests that accurate modeling of beam-cloud interaction requires the kind of continuous model described here. Further work is planned to include external magnetic fields on cloud dynamics and on cloud density variations along the ring.

\section{ACKNOWLEDGMENTS}

This work is supported by USDOE No. DEFG03-93ER40745, No. NSF-PHYS-00787815, No. DE-FC02-01ER41192, No. DE-FG03-92ER40727, No. DE-FC02-01ER41179, and No. PHY-0078508. Computational time and support from the USC HighPerformance-Computing and Communications Center and NERSC are gratefully acknowledged.

\section{APPENDIX: ANALYTIC EXPRESSION FOR THE CLOUD DENSITY COMPRESSION ON THE AXIS OF THE BEAM}

The electron cloud line density $\lambda_{\mathrm{cl}}(z)$ around the beam can be alternatively expressed as a neutralization factor $\eta_{c}(z)$ multiplied by the bunch line density (usually
Gaussian) or in terms of the unperturbed cloud density through multiplication by an appropriate enhancement factor $H_{\mathrm{cl}}(z)$. Note that $\lambda_{\mathrm{cl}}(z)$ is a function of the longitudinal coordinate along the bunch $z$ according to

$$
\lambda_{\mathrm{cl}}(z)=H_{\mathrm{cl}}(z) n_{\mathrm{cl}} e A,
$$

where $A$ is the cross sectional area of the cloud density enhancement on the beam axis, and $n_{\mathrm{cl}}$ is the unperturbed cloud density. The enhancement factor $H_{\mathrm{cl}}$ can be obtained from simulations or an approximate analytically as we show next. A typical profile of the cloud density enhancement along the bunch for CERN-SPS $\left(n_{\mathrm{cl}}=\right.$ $10^{6} \mathrm{~cm}^{-3}, N_{b}=10^{11}$ protons in a Gaussian bunch with $\sigma_{x} \approx \sigma_{y} \cong 2 \mathrm{~mm}, \sigma_{z} \cong 0.3 \mathrm{~m}$, and chamber vertical size $b=4 \mathrm{~cm}$ ) with two different bunch intensities is illustrated in Fig. 11.

An approximate analytic expression for $H_{\mathrm{cl}}$ can be found from the argument below.

The cloud density enhancement is limited by the amount of charge within the vicinity of the beam. By "in the vicinity" we mean within a radius such that it has time to reach the beam axis before the beam passes by.

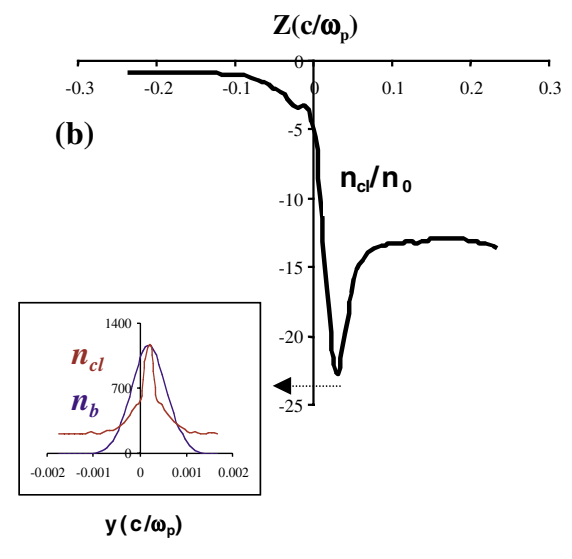

FIG. 11. (Color) Electron cloud line density along the bunch normalized to the unperturbed density for two different values of SPS bunch current. (a) $10^{11} \mathrm{p} / \mathrm{b}$ case. The transverse density profile is taken along the center of the beam (b) $3 \times 10^{10}$ case. The profile is taken at the location of maximum cloud compression which is close to the center of the beam. By definition, these figures show the longitudinal profile of the enhancement factor $H_{\mathrm{cl}}(z)$. In both cases $c / \omega_{p}$ is $5.3 \mathrm{~m}$. 
The time it takes a cloud electron to reach the axis from an original radius $r_{0}$ is given by [23]

$$
t=\frac{\sqrt{\pi}}{\omega_{\mathrm{pb}}} \frac{r_{0}}{\sigma_{r}}
$$

where $\sigma_{r}=\sigma_{x} \cong \sigma_{y}$ is the beam radius (assumed round) and $\omega_{\mathrm{pb}}$ is the electron plasma frequency associated with the beam density (i.e., $\omega_{\mathrm{pb}}^{2}=4 \pi n_{b} e^{2} / m_{e}$ and $n_{b}$ is the peak beam density, $\left.n_{b}=\left[N_{b} /\left(2 \pi \sqrt{2 \pi} \sigma_{x} \sigma_{y} \sigma_{z}\right)\right]\right)$. This analytic result was obtained for a uniform beam profile but proved to be reasonably accurate by comparing it to numerical solutions for Gaussian beams. To effect the core of the bunch, this time must be less than the bunch length $\sigma_{z} / c$. Thus the maximum radius is

$$
\frac{r_{0}}{\sigma_{r}}=\omega_{\mathrm{pb}} \sigma_{z} / c
$$

giving rise to a maximum charge density enhancement

$$
n_{\max } / n_{0}=H_{\mathrm{cl}} \frac{A}{2 \pi \sigma_{r}^{2}}=\left(\frac{r_{0}}{\sigma_{r}}\right)^{2}=\left(\frac{\omega_{\mathrm{pb}} \sigma_{z}}{c}\right)^{2} .
$$

For the example parameters of Fig. 11, this gives $H_{\mathrm{cl}} A / 2 \pi \sigma_{r}^{2}=17$ and 6 , respectively. This compares reasonably with the values 17 and 8.5 obtained from the simulations.

[1] H. Fukuma, K. Cornelis, F.-J. Decker, R. Macek, E. Metral, and W. Fischer, in Proceedings of ECLOUD'02, CERN, Geneva, 2002, edited by G. Rumolo and F. Zimmermann (CERN Yellow Report No. CERN-2002-001, 2002).

[2] G. Rumolo, F. Ruggiero, and F. Zimmermann, Phys. Rev. ST Accel. Beams 4, 012801 (2001).

[3] M. Jimenez et al., in Proceedings of ECLOUD'02, CERN, Geneva, 2002 (Ref. [1]).

[4] A. Rossi, G. Rumolo, and F. Zimmermann, in Proceedings of ECLOUD'O2, CERN, Geneva, 2002 (Ref. [1]).
[5] G. Arduini, K. Cornelis, W. Hoefle, G. Rumolo, and F. Zimmermann, in Proceedings of PAC2001, Chicago (IEEE, Piscataway, NJ, 2001).

[6] K. Oide, in Proceedings of Chamonix XI, 2001 (CERN Report No. SL-2001-003, 2001).

[7] S. S. Win et al., in Proceedings of the second Asian Particle Accelerator Conference, Bejing, 2001 (IHEP, Beijing, 2002).

[8] R. Cappi, M. Giovannozzi, E. Metral, G. Rumolo, and F. Zimmermann, Phys. Rev. ST Accel. Beams 5, 094401 (2002).

[9] K. Ohmi and F. Zimmermann, Phys. Rev. Lett. 85, 3821 (2000).

[10] K. Ohmi, S. Heifets, and F. Zimmermann, CERN Report No. SL-2001-062(AP), 2001.

[11] F. Zimmermann, in Proceedings of Chamonix XI, 2001 (Ref. [6]).

[12] G. Rumolo and F. Zimmermann, in Proceedings of ECLOUD'02, CERN, Geneva, 2002 (Ref. [1]).

[13] M. A. Furman and A. A. Zholents, in Proceedings of PAC99, New York City, 1999 (IEEE, Piscataway, NJ, 1999), p. 1794.

[14] M. Blaskiewicz, M. A. Furman, M. Pivi, and R. J. Macek, Phys. Rev. ST Accel. Beams 6, 014203 (2003).

[15] W. Fischer, J. M. Brennan, M. Blaskiewicz, and T. Satogata, Phys. Rev. ST Accel. Beams 5, 124401 (2002).

[16] F. Zimmermann, CERN LHC Project Report No. 95, SLAC-PUB-7425, 1997.

[17] G. Rumolo and F. Zimmermann, in Proceedings of ECLOUD'02, CERN, Geneva, 2002 (Ref. [1]).

[18] M. T. F. Pivi and M. A. Furman, Phys. Rev. ST Accel. Beams 6, 034201 (2003).

[19] C. Huang et al., in Proceedings of the 18th Annual Review of Progress in Applied Electromagnetics, Monterey, CA, 2002 (to be published), p. 557.

[20] D. H. Whittum, Phys. Plasmas 4, 1154 (1997).

[21] James H. Cooley and Thomas M. Anderson, Jr., in Advanced Accelerator Concepts, Tenth Workshop, edited by Christopher E. Clayton and Patrick Muggli, AIP Conf. Proc. No. 647 (AIP, New York, 2002), p. 232.

[22] V. K. Decyk and J. E. Slottow, Comput. Phys., 3, 50 (1989).

[23] S. Lee et al., Phys. Rev. E 64, 045501 (2001). 